\title{
Families of sets not belonging to algebras and combinatorics of finite sets of ultrafilters
}

\section{Leonid Š Grinblat ${ }^{*}$}

\section{"Correspondence:} grinblat@ariel.ac.il

Department of Computer Science and Mathematics, Ariel University, Ariel, Israel

\begin{abstract}
This article is a part of the theory developed by the author in which the following problem is solved under natural assumptions: to find necessary and sufficient conditions under which the union of at most countable family of algebras on a certain set $X$ is equal to $\mathcal{P}(X)$. Here the following new result is proved. Let $\left\{\mathcal{A}_{\lambda}\right\}_{\lambda \in \Lambda}$ be a finite collection of algebras of sets given on a set $X$ with $\#(\Lambda)=n>0$, and for each $\lambda$ there exist at least $\frac{10}{3} n+\sqrt{\frac{2 n}{3}}$ pairwise disjoint sets belonging to $\mathcal{P}(X) \backslash \mathcal{A}_{\lambda}$. Then there exists a family $\left\{U_{\lambda^{\prime}}^{1}, U_{\lambda}^{2}\right\}_{\lambda \in \Lambda}$ of pairwise disjoint subsets of $X\left(U_{\lambda}^{i} \cap U_{\lambda^{\prime}}^{j}=\emptyset\right.$ except the case $\left.\lambda=\lambda^{\prime}, i=j\right)$; and for each $\lambda$ the following holds: if $Q \in \mathcal{P}(X)$ and $Q$ contains one of the two sets $U_{\lambda}^{1}, U_{\lambda}^{2}$ and its intersection with the other set is empty, then $Q \notin \mathcal{A}_{\lambda}$.

MSC: Primary 03E05; secondary 54D35
\end{abstract}

Keywords: algebra of sets; $\sigma$-algebra; ultrafilter; pairwise disjoint sets

\section{Introduction}

The present article is a further development of the theory formulated in [1-7]. The topic studied in these articles, as well as in the present paper, is sets not belonging to algebras of sets.

Definition 1.1 An algebra $\mathcal{A}$ on a set $X$ is a non-empty family of subsets of $X$ possessing the following properties: (1) if $M \in \mathcal{A}$, then $X \backslash M \in \mathcal{A}$; (2) if $M_{1}, M_{2} \in \mathcal{A}$, then $M_{1} \cup M_{2} \in \mathcal{A}$.

It is clear that if $M_{1}, M_{2} \in \mathcal{A}$, then $M_{1} \cap M_{2} \in \mathcal{A}$ and $M_{1} \backslash M_{2} \in \mathcal{A}$; also, it is clear that $X \in \mathcal{A}$.

\subsection{Some notation and names}

All algebras and measures are considered on some abstract set $X \neq \emptyset$. When it is clear from the context, we will not state explicitly that a set belongs to the family $\mathcal{P}(X)$ of all subsets of $X$. By $\mathbb{N}^{+}$we denote the set of natural numbers. If $n_{1}, n_{2} \in \mathbb{N}^{+}$and $n_{1} \leq n_{2}$, then $\left[n_{1}, n_{2}\right]=\left\{k \in \mathbb{N}^{+} \mid n_{1} \leq k \leq n_{2}\right\}$. Let $\rho$ be a real number. By $\lfloor\rho\rfloor$ we denote the maximum integer $\leq \rho$. By $\lceil\rho\rceil$ we denote the minimum integer $\geq \rho$. The symbol \# $(M)$ denotes the cardinality of the set $M$. A set $M$ is countable if $\#(M)=\aleph_{0}$.

The following concept was used in [5].

Definition 1.2 An algebra $\mathcal{A}$ has $\kappa$ lacunae, where $\kappa$ is a cardinal number, if there exist $\kappa$ pairwise disjoint sets not belonging to $\mathcal{A}$.

o 2015 Grinblat; licensee Springer. This is an Open Access article distributed under the terms of the Creative Commons Attribution License (http://creativecommons.org/licenses/by/4.0), which permits unrestricted use, distribution, and reproduction in any medium, provided the original work is properly credited. 
Let $\left\{\mathcal{A}_{\lambda}\right\}_{\lambda \in \Lambda}$ be a family of algebras and $\mathcal{A}_{\lambda} \neq \mathcal{P}(X)$ for each $\lambda \in \Lambda$. The following natural question arises: what are possible conditions that distinguish between the cases $\bigcup_{\lambda \in \Lambda} \mathcal{A}_{\lambda} \neq \mathcal{P}(X)$ and $\bigcup_{\lambda \in \Lambda} \mathcal{A}_{\lambda}=\mathcal{P}(X)$ ? Let $\#(\Lambda) \leq \aleph_{0}$, and let us assume that $\mathcal{A}_{\lambda}$ are $\sigma$ algebras if $\#(\Lambda)=\aleph_{0}$. In [6] we obtained necessary and sufficient conditions for the equality $\bigcup_{\lambda \in \Lambda} \mathcal{A}_{\lambda}=\mathcal{P}(X)$ to hold. The first publication connected with this topic was that of Erdös [8] (this paper contains the well-known theorem of Alouglu-Erdös). Some information about the history of the subject after the publication of [8] and before the publication of [1] is presented in [2]. In fact, Alouglu and Erdös studied non-measurable sets with respect to families of measures. Let $\aleph_{1} \leq \#(X) \leq 2^{\aleph_{0}}$. Let a $\sigma$-additive measure $\mu$ be defined on $X$. Here $\mu(X)=1$, the measure of a one-point set equals 0 , and the measure of each $\mu$-measurable set equals 0 or 1 . Such a measure $\mu$ is called a $\sigma$-two-valued measure. Clearly, there exist $\mu$-non-measurable sets. The Alouglu-Erdös theorem states that if $\#(X)=\aleph_{1}$, then for any countable family of $\sigma$-two-valued measures $\mu_{1}, \ldots, \mu_{k}, \ldots$ there exists a set which is non-measurable with respect to all these measures. The proof of the Alouglu-Erdös theorem is very simple and is based on the possibility of constructing the well-known Ulam matrix (see [9]). The non-trivial Gitik-Shelah theorem (see [10]) asserts the validity of the Alouglu-Erdös theorem if $\#(X)=2^{\aleph_{0}}$. Obviously, the Gitik-Shelah theorem is a generalization of the Alouglu-Erdös theorem. The Gitik-Shelah theorem can be reformulated in our language. As before, let us consider the $\sigma$-two-valued measures $\mu_{1}, \ldots, \mu_{k}, \ldots$. For each measure $\mu_{k}$, we examine the algebra $\mathcal{A}_{k}$ of all $\mu_{k}$ measurable sets. The Gitik-Shelah theorem asserts that $\bigcup_{k \in \mathbb{N}^{+}} \mathcal{A}_{k} \neq \mathcal{P}(X)$. We note that here each algebra $\mathcal{A}_{k}$ has $\aleph_{0}$ lacunae. If $\#(X)=\aleph_{1}$, then the situation is much simpler: each algebra $\mathcal{A}_{k}$ has $\aleph_{1}$ lacunae. The Gitik-Shelah theorem is used in the proofs of our theorems for countable families of $\sigma$-algebras.

Definition 1.3 Let $\left\{\mathcal{A}_{\lambda}\right\}_{\lambda \in \Lambda}$ be a family of algebras, and $\left\{U_{\lambda}^{1}, U_{\lambda}^{2}\right\}_{\lambda \in \Lambda}$ be a family of sets with the following properties:

(1) $U_{\lambda}^{i} \cap U_{\lambda^{\prime}}^{j}=\emptyset$ except when $\lambda=\lambda^{\prime}, i=j$;

(2) for any $\lambda \in \Lambda$, the following holds: if a set $Q$ contains one of the two sets $U_{\lambda}^{1}, U_{\lambda}^{2}$ and its intersection with the other set is empty, then $Q \notin \mathcal{A}_{\lambda}$.

Then we say that the family $\left\{\mathcal{A}_{\lambda}\right\}_{\lambda \in \Lambda}$ has the full set of lacunae $\left\{U_{\lambda}^{1}, U_{\lambda}^{2}\right\}_{\lambda \in \Lambda}$.

Now we give a simple proposition.

Proposition 1.4 If a family of algebras $\left\{\mathcal{A}_{\lambda}\right\}_{\lambda \in \Lambda}$ has the full set of lacunae $\left\{U_{\lambda}^{1}, U_{\lambda}^{2}\right\}_{\lambda \in \Lambda}$, then there exists a family of pairwise distinct sets $\left\{Q_{\vartheta}\right\}_{\vartheta \in \Theta}$ such that the following holds:

(1) $Q_{\vartheta} \notin \bigcup_{\lambda \in \Lambda} \mathcal{A}_{\lambda}$ for any $\vartheta \in \Theta$;

(2) any set $Q_{\vartheta}$ is a union of sets $U_{\lambda}^{i}$;

(3) $Q_{\theta_{1}} \backslash Q_{\vartheta_{2}} \notin \bigcap_{\lambda \in \Lambda} \mathcal{A}_{\lambda}$ for any pair $\vartheta_{1} \neq \vartheta_{2}$;

(4) $\#(\Theta)=2^{\#(\Lambda)}$.

Proof Put $\Theta=\mathcal{P}(\Lambda)$. If $\vartheta \in \mathcal{P}(\Lambda)$, put

$$
Q_{\vartheta}=\left(\bigcup_{\lambda \in \vartheta} U_{\lambda}^{1}\right) \cup\left(\bigcup_{\lambda \in \Lambda \backslash \vartheta} U_{\lambda}^{2}\right) .
$$


In this paper we deal mostly with the following problem: under which conditions a family of algebras $\left\{\mathcal{A}_{\lambda}\right\}_{\lambda \in \Lambda}$ has a full set of lacunae. We assume that $\#(\Lambda) \leq \aleph_{0}$. This was studied in [1-3]. The proof of the two following theorems can be found in [2].

Theorem 1.5 Let $\mathcal{A}_{1}, \ldots, \mathcal{A}_{n}$ be a finite family of algebras, and assume that for each $k \in$ $[1, n]$ the algebra $\mathcal{A}_{k}$ has $4 k-3$ lacunae. Then this family has a full set of lacunae.

It is easy to prove (see [2], Chapter 14) that the estimate $4 k-3$ is the best possible in some sense.

Theorem 1.6 Let $\left\{\mathcal{A}_{k}\right\}_{k \in \mathbb{N}^{+}}$be a family of $\sigma$-algebras, and assume that for each $k$ the algebra $\mathcal{A}_{k}$ has $4 k-3$ lacunae. Then this family has some full set of lacunae.

Remark 1.7 Using the notion of absolute introduced by Gleason in [11], we can construct a family of algebras $\left\{\mathcal{B}_{k}\right\}_{k \in \mathbb{N}^{+}}$with the following properties: each algebra $\mathcal{B}_{k}$ has $\aleph_{0}$ lacunae, is not a $\sigma$-algebra, and $\bigcup_{k \in \mathbb{N}^{+}} \mathcal{B}_{k}=\mathcal{P}(X)$ (see [2], Chapter 5). Hence, Theorem 1.6 and Theorem 2.4 below do not hold if we claim them for algebras which are not assumed to be $\sigma$-additive. Therefore, we suppose that all algebras of a countable family of algebras are $\sigma$-algebras.

The following definition was given in [2].

Definition 1.8 For each $n \in \mathbb{N}^{+}$, denote by $\mathfrak{v}(n)$ the minimal cardinal number such that if $\left\{\mathcal{A}_{\lambda}\right\}_{\lambda \in \Lambda}, \#(\Lambda)=n$, is a family of algebras, and for each $\lambda \in \Lambda$ the algebra $\mathcal{A}_{\lambda}$ has $\mathfrak{v}(n)$ lacunae, then the family $\left\{\mathcal{A}_{\lambda}\right\}_{\lambda \in \Lambda}$ has a full set of lacunae.

In [2] we proved that:

(1) $\mathfrak{v}(n)=4 n-3$ for $n \leq 3$;

(2) $\mathfrak{v}(n) \leq 4 n-5$ for $n>3$;

(3) $\mathfrak{v}(n) \leq 4 n-\left\lfloor\frac{n+3}{2}\right\rfloor$ for any $n$;

(4) $3 n-2 \leq \mathfrak{v}(n)$ for any $n$.

In this paper we will improve the upper bound of $\mathfrak{v}(n)$.

From here until the end of Section 1 we present propositions and notions which form the method of proofs of our theorems. This method first appeared in [1] and was later used in [2-7]. Let $\beta X$ be the Stone-Čech compactification of $X$ with the discrete topology; $\beta X$ is the family of all ultrafilters on $X$.

Consider an algebra $\mathcal{A}$. We say that $a, b \in \beta X$ are $\mathcal{A}$-equivalent iff $a \cap \mathcal{A}=b \cap \mathcal{A}$. Let $[b]_{\mathcal{A}}$ denote the $\mathcal{A}$-equivalence class of $b$, and define the kernel of the algebra $\mathcal{A}$ :

$$
\operatorname{ker} \mathcal{A}=\left\{b \in \beta X \mid \#\left([b]_{\mathcal{A}}\right)>1\right\}
$$

If $\mathcal{A}=\mathcal{P}(X)$, then $\operatorname{ker} \mathcal{A}=\emptyset$. From now on, when we say $a$ and $b$ are $\mathcal{A}$-equivalent ultrafilters, we always assume that $a \neq b$. If $a, b$ are $\mathcal{A}$-equivalent ultrafilters, then we say that $a$ has an $\mathcal{A}$-equivalent ultrafilter $b$, or $a$ is $\mathcal{A}$-equivalent to $b$.

Statement 1.9 Consider an algebra $\mathcal{A}$ and sets $U, V \in \mathcal{P}(X)$ such that $U \cap V=\emptyset$. The following two conditions are equivalent. (1) Each set $Q$ containing one of the sets $U, V$ and 
being disjoint from the other does not belong to $\mathcal{A}$. (2) There exist $\mathcal{A}$-equivalent ultrafilters $a, b$ such that $U \in a, V \in b$.

Proof It is obvious that (1) follows from (2). Let us prove that (2) follows from (1). Let us assume the contrary. We fix an ultrafilter $q \ni U$. For any ultrafilter $r \ni V$, we choose a set $W(r) \in r$ such that $W(r) \in \mathcal{A}$ and $W(r) \notin q$. Since the set of all ultrafilters which contain $V$ is a compact subset of $\beta X$, there exists a finite sequence of sets $W\left(r_{1}\right), \ldots, W\left(r_{m}\right)$ with the following properties:

(1) $W\left(r_{k}\right) \in \mathcal{A}$ for any $k \in[1, m]$

(2) $W\left(r_{k}\right) \notin q$ for any $k \in[1, m]$;

(3) $V \subseteq \bigcup_{k=1}^{m} W\left(r_{k}\right)$.

Let

$$
\widetilde{W}(q)=X \backslash \bigcup_{k=1}^{m} W\left(r_{k}\right) .
$$

It is clear that $\widetilde{W}(q) \in q, \widetilde{W}(q) \in \mathcal{A}$, and $\widetilde{W}(q) \cap V=\emptyset$. Since the set of all ultrafilters which contain $U$ is a compact subset of $\beta X$, there exists a finite sequence of sets $\widetilde{W}\left(q_{1}\right), \ldots, \widetilde{W}\left(q_{n}\right)$ such that $\widetilde{W}\left(q_{k}\right) \in \mathcal{A}$ for any $k \in[1, n], \bigcup_{k=1}^{n} \widetilde{W}\left(q_{k}\right)=\widetilde{W} \supseteq U$, and $\widetilde{W} \cap V=\emptyset$. We have $\widetilde{W} \in \mathcal{A}$, a contradiction.

The following crucial claim is a direct consequence of Statement 1.9.

Claim 1.10 Consider an algebra $\mathcal{A}$ and $U \in \mathcal{P}(X)$. Then $U \notin \mathcal{A}$ if and only if there exist $\mathcal{A}$-equivalent ultrafilters $p$ and $q$ such that $U \in p$ and $U \notin q$.

Proof The sufficiency is obvious. If $U \notin \mathcal{A}$, then the sets $U$ and $V=X \backslash U$ satisfy the condition (1) of Statement 1.9. Therefore, there exist the corresponding ultrafilters $p$ and $q$.

It is clear that if $\mathcal{A} \neq \mathcal{P}(X)$, then $\#(\operatorname{ker} \mathcal{A}) \geq 2$. It is rather easy to show that an algebra $\mathcal{A}$ has $k$ lacunae, where $2 \leq k \leq \aleph_{0}$, if and only if $\#(\operatorname{ker} \mathcal{A}) \geq k^{\text {a }}$

Definition 1.11 A set $M \subseteq \beta X$ is said to be $\mathcal{A}$-equivalent if $\#(M)>1$, any two distinct ultrafilters in $M$ are $\mathcal{A}$-equivalent, and there exist no $\mathcal{A}$-equivalent ultrafilters $a, b$ such that $a \in M, b \notin M$.

Obviously, an $\mathcal{A}$-equivalent set has the form $[b]_{\mathcal{A}}$ (see above). Also it is obvious that an $\mathcal{A}$-equivalent set is closed in $\beta X$.

Remark 1.12 Consider algebras $\mathcal{A}, \mathcal{B}$. It is very easy to prove that the following statements are equivalent.

(1) $\mathcal{A} \supseteq \mathcal{B}$.

(2) If $a$ and $b$ are $\mathcal{A}$-equivalent ultrafilters, then $a$ and $b$ are $\mathcal{B}$-equivalent ultrafilters.

(3) If $M$ is an $\mathcal{A}$-equivalent set, then $M$ is contained in a certain $\mathcal{B}$-equivalent set.

Remark 1.13 If $M \subseteq \beta X$ (in particular, if $M \subseteq X$ ), then by $\bar{M}$ we denote the closure $M$ in $\beta X$. The following arguments will be used later in this paper. Let $A \subseteq \beta X, 2 \leq \#(A)<\aleph_{0}$. 
The set $A$ is divided into pairwise disjoint sets $A_{1}, \ldots, A_{m}$ and $\#\left(A_{k}\right)>1$ for each $k \in[1, m]$. Two different ultrafilters are called $a$-equivalent if and only if they belong to the same set $A_{k}$. We can construct the algebra $\mathcal{A}$ such that the $a$-equivalence relation is in fact the $\mathcal{A}$-equivalence relation, $\operatorname{ker} \mathcal{A}=A$, and $A_{1}, \ldots, A_{m}$ are all $\mathcal{A}$-equivalent sets. Indeed, by definition $M \in \mathcal{A}$ if and only if for each $k \in[1, m]$ either $A_{k} \cap \bar{M}=\emptyset$, or $A_{k} \subseteq \bar{M}$.

Remark 1.14 Let us recall that an algebra which does not have $\aleph_{0}$ lacunae is called $\omega$ saturated. So, an algebra $\mathcal{A}$ is $\omega$-saturated if and only if $\#(\operatorname{ker} \mathcal{A})<\aleph_{0}$. The algebra $\mathcal{A}$ from Remark 1.13 is $\omega$-saturated.

Remark 1.15 Further we use two following very simple statements. (1) By Statement 1.9 a finite family of algebras $\mathcal{A}_{1}, \ldots, \mathcal{A}_{n}$ has a full set of lacunae if and only if there exist $2 n$ pairwise distinct ultrafilters $a_{1}, \ldots, a_{n}, b_{1}, \ldots, b_{n}$ such that $a_{k}, b_{k}$ are $\mathcal{A}_{k}$-equivalent ultrafilters for each $k \in[1, n]$. (2) Let $\mathfrak{A}=\left\{\mathcal{A}_{\lambda}\right\}_{\lambda \in \Lambda}$ and $\mathfrak{A}^{\prime}=\left\{\mathcal{A}_{\lambda}^{\prime}\right\}_{\lambda \in \Lambda}$ be two non-empty families of algebras, and $\mathcal{A}_{\lambda}^{\prime} \supseteq \mathcal{A}_{\lambda}$ for every $\lambda \in \Lambda$. Assume that the family $\mathfrak{A}^{\prime}$ has a full set of lacunae $\left\{U_{\lambda}^{1}, U_{\lambda}^{2}\right\}_{\lambda \in \Lambda}$. Then the family $\mathfrak{A}$ has the same full set of lacunae $\left\{U_{\lambda}^{1}, U_{\lambda}^{2}\right\}_{\lambda \in \Lambda}$.

\section{Main results. An open problem}

The following result was announced in [3]: $\mathfrak{v}(n) \leq\left\lceil\frac{10}{3} n+\frac{2}{\sqrt{3}} \sqrt{n}\right\rceil$ for any $n$. In this paper a stronger theorem is proved.

Theorem 2.1 $\mathfrak{v}(n) \leq\left\lceil\frac{10}{3} n+\sqrt{\frac{2 n}{3}}\right\rceil$.

Remark 2.2 The combinatorial nature of Theorem 2.1 is discussed in Section 4. Also in Section 4 the proof of Theorem 4.5 uses the classical Ramsey theorem.

Problem 2.3 We know that $\mathfrak{v}(n) \geq 3 n-2$ for any $n$, and $\mathfrak{v}(n)>3 n-2$ if $n=2,3$ since $\mathfrak{v}(2)=5, \mathfrak{v}(3)=9$ (see Section 1). Is it true that $\mathfrak{v}(n)=3 n-2$ for any $n \neq 2,3$ ? This result is obviously true for $n=1$.

The final section of this article is devoted to the proof of the following theorem, which is a generalization of theorems of Alaouglu-Erdös and Gitik-Shelah.

Theorem 2.4 It is possible to construct nondecreasing functions $\varphi: \mathbb{N}^{+} \rightarrow \mathbb{N}^{+}$such that the following conditions hold:

(1) $\underline{\lim }_{n \rightarrow \infty} \frac{\varphi(n)-\frac{10}{3} n}{\sqrt{n}}=\sqrt{\frac{2}{3}}$;

(2) if $\left\{\mathcal{A}_{k}\right\}_{k \in \mathbb{N}^{+}}$is a family of $\sigma$-algebras and each algebra $\mathcal{A}_{k}$ has $\varphi(k)$ lacunae, then this family has a full set of lacunae.

\section{Finite families of algebras. Proof of Theorem 2.1}

The following lemma is used in the proof of Lemma 3.2.

Lemma 3.1 Consider an algebra $\mathcal{A}$ which is not $\omega$-saturated; $;$ let a number $\xi \in \mathbb{N}^{+}$be given. Then it is possible to construct an $\omega$-saturated algebra $\mathcal{A}^{\prime}$ such that $\#\left(\operatorname{ker} \mathcal{A}^{\prime}\right) \geq \xi$ and $\mathcal{A}^{\prime} \supset \mathcal{A}$. 
Proof Take two distinct $\mathcal{A}$-equivalent ultrafilters $s_{1}, t_{1}$. Consider two distinct ultrafilters $a_{1}, a_{2} \in \operatorname{ker} \mathcal{A} \backslash\left\{s_{1}, t_{1}\right\}$. If $a_{1}$ has an $\mathcal{A}$-equivalent ultrafilter in $\left\{s_{1}, t_{1}\right\}$, and $a_{2}$ has an $\mathcal{A}$ equivalent ultrafilter in $\left\{s_{1}, t_{1}\right\}$, then $a_{1}$ and $a_{2}$ are $\mathcal{A}$-equivalent ultrafilters. Denote $s_{2}=a_{1}$, $t_{2}=a_{2}$. If, for example, $a_{1}$ does not have an $\mathcal{A}$-equivalent ultrafilter in $\left\{s_{1}, t_{1}\right\}$, then take an ultrafilter $c$ such that $a_{1} \neq c$ and $a_{1}, c$ are $\mathcal{A}$-equivalent ultrafilters. In this case denote $s_{2}=a_{1}, t_{2}=c$. Now take three pairwise disjoint ultrafilters $b_{1}, b_{2}, b_{3} \in \operatorname{ker} \mathcal{A} \backslash\left\{s_{1}, t_{1}, s_{2}, t_{2}\right\}$. If every ultrafilter $b_{i}$ has an $\mathcal{A}$-equivalent ultrafilter in $\left\{s_{1}, t_{1}, s_{2}, t_{2}\right\}$, then in the set $\left\{b_{1}, b_{2}, b_{3}\right\}$ we can choose two distinct $\mathcal{A}$-equivalent ultrafilters, for example, $b_{1}$ and $b_{2}$. Put $s_{3}=b_{1}$, $t_{3}=b_{2}$. If, for example, $b_{1}$ does not have an $\mathcal{A}$-equivalent ultrafilter in $\left\{s_{1}, t_{1}, s_{2}, t_{2}\right\}$, then take an ultrafilter $d$ such that $b_{1} \neq d$ and $b_{1}, d$ are $\mathcal{A}$-equivalent ultrafilters. Denote $s_{3}=b_{1}$, $t_{3}=d$. It is clear that for every $\ell \in \mathbb{N}^{+}$it is possible to construct a sequence of pairwise distinct ultrafilters $s_{1}, t_{1}, \ldots, s_{\ell}, t_{\ell}$ such that $s_{i}$ and $t_{i}$ are $\mathcal{A}$-equivalent ultrafilters for all $i \in[1, \ell]$. Let $2 \ell \geq \xi$. Define $M_{1}=\left\{s_{1}, t_{1}\right\}, \ldots, M_{\ell}=\left\{s_{\ell}, t_{\ell}\right\}$. By Remark 1.13 it is possible to construct an algebra $\mathcal{A}^{\prime}$ such that $\operatorname{ker} \mathcal{A}^{\prime}=\bigcup_{i=1}^{\ell} M_{i}$ and $M_{1}, \ldots, M_{\ell}$ are $\mathcal{A}^{\prime}$-equivalent sets.

The following lemma is given in [2] without proof.

Lemma $3.2 \mathfrak{v}(n) \in \mathbb{N}^{+}$, and $\mathfrak{v}(n+1)-\mathfrak{v}(n) \leq 4$.

Proof It is obvious that $\mathfrak{v}(1)=1$. Let $n \in \mathbb{N}^{+}$and assume that $\mathfrak{v}(n) \in \mathbb{N}^{+}$. Consider a family of algebras $\mathcal{A}_{1}, \ldots, \mathcal{A}_{n+1}$ with $\#\left(\operatorname{ker} \mathcal{A}_{k}\right) \geq \mathfrak{v}(n)+4$ for each $k \in[1, n+1]$. We must prove that this family has a full set of lacunae. By Lemma 3.1 and the arguments in Remark 1.15 we can assume that the algebras $\mathcal{A}_{1}, \ldots, \mathcal{A}_{n+1}$ are $\omega$-saturated. We choose $\mathcal{A}_{n+1}$-equivalent ultrafilters $s_{n+1}^{(1)}, s_{n+1}^{(2)}$. Put $B_{k}=\operatorname{ker} \mathcal{A}_{k} \backslash\left\{s_{n+1}^{(1)}, s_{n+1}^{(2)}\right\}$ for each $k \in[1, n]$. Put

$$
\begin{aligned}
B_{k}^{\prime}= & \left\{q \in B_{k} \mid q \text { does not have an } A_{k} \text {-equivalent ultrafilter in } B_{k}\right. \\
& \text { and has an } \left.A_{k} \text {-equivalent ultrafilter in }\left\{s_{n+1}^{(1)}, s_{n+1}^{(2)}\right\}\right\} .
\end{aligned}
$$

It is clear that $\#\left(B_{k}^{\prime}\right) \leq 2$. Put $B_{k}^{\prime \prime}=B_{k} \backslash B_{k}^{\prime}$. Clearly, each ultrafilter in $B_{k}^{\prime \prime}$ has an $\mathcal{A}_{k}$ equivalent ultrafilter in $B_{k}^{\prime \prime}$. Therefore, by Remark 1.13, we can construct an algebra $\mathcal{A}_{k}^{\prime}$ such that $\operatorname{ker} \mathcal{A}_{k}^{\prime}=B_{k}^{\prime \prime}$ and the $\mathcal{A}_{k}^{\prime}$-equivalent relation in $\operatorname{ker} \mathcal{A}_{k}^{\prime}$ is in fact the $\mathcal{A}_{k}$-equivalent relation. We have $\#\left(\operatorname{ker} \mathcal{A}_{k}^{\prime}\right) \geq \mathfrak{v}(n)$ for each $k \in[1, n]$. Therefore, there exist $2 n$ pairwise distinct ultrafilters $s_{1}^{(1)}, s_{1}^{(2)}, \ldots, s_{n}^{(1)}, s_{n}^{(2)}$, and $s_{k}^{(1)}, s_{k}^{(2)}$ are $\mathcal{A}_{k}$-equivalent ultrafilters from $\operatorname{ker} \mathcal{A}_{k}^{\prime}$. We have pairwise distinct ultrafilters $s_{1}^{(1)}, s_{1}^{(2)}, \ldots, s_{n+1}^{(1)}, s_{n+1}^{(2)}$, and $s_{k}^{(1)}, s_{k}^{(2)}$ are $\mathcal{A}_{k}$-equivalent ultrafilters for each $k \in[1, n+1]$.

Remark 3.3 It is obvious that $\mathfrak{v}(1)=1$. Therefore, by Lemma 3.2 we have $\mathfrak{v}(n) \leq 4 n-3$ for any $n$. In Chapter 14, [2], we proved that $\mathfrak{v}(4) \leq 11$. Therefore, by Lemma 3.2, we have that $\mathfrak{v}(n) \leq 4 n-5$ for any $n \geq 4$.

We now turn to the proof of Theorem 2.1. This proof is a strong improvement of the proposition $\mathfrak{v}(n) \leq 4 n-\left\lfloor\frac{n+3}{2}\right\rfloor$ mentioned above (see [2], Chapter 14). 
Proof of Theorem 2.1 (1) By Remark 3.3 our theorem is true for all $n \leq 13$. (This can be verified by a simple computation.) Fix a natural number $n \geq 14$ and a real number

$$
\omega(n) \geq \sqrt{\frac{2 n}{3}}
$$

Let $\mathcal{A}_{1}, \ldots, \mathcal{A}_{n}$ be algebras such that

$$
\#\left(\operatorname{ker} \mathcal{A}_{k}\right) \geq \frac{10}{3} n+\omega(n)
$$

for each $k \in[1, n]$. By Lemma 3.1 and arguments in Remark 1.15, we can assume that the algebras $\mathcal{A}_{1}, \ldots, \mathcal{A}_{n}$ are $\omega$-saturated. We will prove that there exist pairwise distinct ultrafilters

$$
a_{1}^{*}, \ldots, a_{n}^{*}, b_{1}^{*}, \ldots, b_{n}^{*}
$$

such that $a_{k}^{*}, b_{k}^{*}$ are $\mathcal{A}_{k}$-equivalent ultrafilters for each $k \in[1, n]$. Our goal is to contradict the assumption that ultrafilters $a_{1}^{*}, \ldots, a_{n}^{*}, b_{1}^{*}, \ldots, b_{n}^{*}$ do not exist. Inductively assume that

$$
\mathfrak{v}(n-1) \leq\left\lceil\frac{10}{3}(n-1)+\sqrt{\frac{2 n-2}{3}}\right\rceil \text {. }
$$

Then there exists a set of pairwise distinct ultrafilters

$$
\mathfrak{F}=\left\{a_{1}, \ldots, a_{n-1}, b_{1}, \ldots, b_{n-1}\right\}
$$

such that $a_{k}, b_{k}$ are $\mathcal{A}_{k}$-equivalent ultrafilters for each $k \in[1, n-1]$. Consider $\operatorname{ker} \mathcal{A}_{n}$. It is clear that

$$
\#\left(\operatorname{ker} \mathcal{A}_{n} \backslash \mathfrak{F}\right) \geq \frac{10}{3} n+\omega(n)-2 n+2=\frac{4}{3} n+\omega(n)+2
$$

If there exist two $\mathcal{A}_{n}$-equivalent ultrafilters in $\operatorname{ker} \mathcal{A}_{n} \backslash \mathfrak{F}$, we immediately obtain the required construction yielding the existence of ultrafilters $a_{1}^{*}, \ldots, a_{n}^{*}, b_{1}^{*}, \ldots, b_{n}^{*}$. Therefore, each ultrafilter from $\operatorname{ker} \mathcal{A}_{n} \backslash \mathfrak{F}$ has an $\mathcal{A}_{n}$-equivalent ultrafilter in $\mathfrak{F}$. Therefore, there exist distinct ultrafilters $c_{n}, d_{n} \in \operatorname{ker} \mathcal{A}_{n} \backslash \mathfrak{F}$ and $k_{1} \in[1, n-1]$ such that $\left(a_{k_{1}}, c_{n}\right)$ and $\left(b_{k_{1}}, d_{n}\right)$ are two pairs of $\mathcal{A}_{n}$-equivalent ultrafilters. For simplicity, say $k_{1}=1$. Now consider $\operatorname{ker} \mathcal{A}_{1}$. It is clear that

$$
\#\left(\operatorname{ker} \mathcal{A}_{1} \backslash\left(\mathfrak{F} \cup\left\{c_{n}, d_{n}\right\}\right)\right) \geq \frac{10}{3} n+\omega(n)-2 n=\frac{4}{3} n+\omega(n)
$$

If there exist two $\mathcal{A}_{1}$-equivalent ultrafilters in $\operatorname{ker} \mathcal{A}_{1} \backslash\left(\mathfrak{F} \cup\left\{c_{n}, d_{n}\right\}\right)$, we immediately obtain the required construction yielding the existence of ultrafilters $a_{1}^{*}, \ldots, a_{n}^{*}, b_{1}^{*}, \ldots, b_{n}^{*}$. Similarly, if an ultrafilter in $\operatorname{ker} \mathcal{A}_{1} \backslash\left(\mathfrak{F} \cup\left\{c_{n}, d_{n}\right\}\right)$ has an $\mathcal{A}_{1}$-equivalent ultrafilter in $\left\{a_{1}, b_{1}, c_{n}, d_{n}\right\}$, then the construction which contradicts the non-existence of ultrafilters $a_{1}^{*}, \ldots, a_{n}^{*}, b_{1}^{*}, \ldots, b_{n}^{*}$ is yielded immediately. So, each ultrafilter in $\operatorname{ker} \mathcal{A}_{1} \backslash\left(\mathfrak{F} \cup\left\{c_{n}, d_{n}\right\}\right)$ has an $\mathcal{A}_{1}$-equivalent ultrafilter in the set $\mathfrak{F} \backslash\left\{a_{1}, b_{1}\right\}$. Therefore, there exist distinct ultrafilters $c_{1}, d_{1} \in \operatorname{ker} \mathcal{A}_{1} \backslash\left(\mathfrak{F} \cup\left\{c_{n}, d_{n}\right\}\right)$ and $k_{2} \in[2, n-1]$ such that $\left(a_{k_{2}}, c_{1}\right)$ and $\left(b_{k_{2}}, d_{1}\right)$ are two 
pairs of $\mathcal{A}_{1}$-equivalent ultrafilters. For simplicity, say $k_{2}=2$. This process can be continued. Suppose that there exists a natural number $\eta$ such that

$$
3 \leq \eta \leq \frac{1}{3} n+\omega(n)+2 .
$$

Suppose also that there exists a set of pairwise distinct ultrafilters

$$
\mathfrak{E}=\left\{c_{1}, \ldots, c_{\eta-1}, c_{n}, d_{1}, \ldots, d_{\eta-1}, d_{n}\right\}
$$

and the following holds:

(A) $\left(a_{i+1}, c_{i}\right)$ and $\left(b_{i+1}, d_{i}\right)$ are two pairs of $\mathcal{A}_{i}$-equivalent ultrafilters for each $i \in[2, \eta-1]$;

(B) $\mathfrak{F} \cap \mathfrak{E}=\emptyset$.

Let us recall what we have said above: $\left(a_{1}, c_{n}\right)$ and $\left(b_{1}, d_{n}\right)$ are two pairs of $\mathcal{A}_{n}$-equivalent ultrafilters; $\left(a_{2}, c_{1}\right)$ and $\left(b_{2}, d_{1}\right)$ are two pairs of $\mathcal{A}_{1}$-equivalent ultrafilters.

Define $L_{\eta}=\operatorname{ker} \mathcal{A}_{\eta} \backslash(\mathfrak{F} \cup \mathfrak{E})$. It is clear that

$$
\#\left(L_{\eta}\right) \geq \frac{10}{3} n+\omega(n)-(2 n-2)-2 \eta=\frac{4}{3} n+\omega(n)-2 \eta+2 .
$$

If there exist two $\mathcal{A}_{\eta}$-equivalent ultrafilters in $L_{\eta}$, we immediately obtain the required construction yielding the existence of ultrafilters $a_{1}^{*}, \ldots, a_{n}^{*}, b_{1}^{*}, \ldots, b_{n}^{*}$. Similarly, if an ultrafilter in $L_{\eta}$ has an $\mathcal{A}_{\eta}$-equivalent ultrafilter in $\left\{a_{1}, \ldots, a_{\eta}, b_{1}, \ldots, b_{\eta}\right\} \cup \mathfrak{E}$, then the construction which contradicts the non-existence of ultrafilters $a_{1}^{*}, \ldots, a_{n}^{*}, b_{1}^{*}, \ldots, b_{n}^{*}$ is yielded immediately. Therefore, every ultrafilter from $L_{\eta}$ has an $\mathcal{A}_{\eta}$-equivalent ultrafilter in $\left\{a_{\eta+1}, \ldots, a_{n-1}, b_{\eta+1}, \ldots, b_{n-1}\right\}$. We have

$$
\#\left(L_{\eta}\right)-\#([\eta+1, n-1]) \geq \frac{4}{3} n+\omega(n)-2 \eta+2-n+\eta+1=\frac{1}{3} n+\omega(n)+3-\eta>0 .
$$

Therefore, there exist distinct ultrafilters $c_{\eta}, d_{\eta} \in L_{\eta}$ and $k_{\eta+1} \in[\eta+1, n-1]$ such that $\left(a_{k_{\eta+1}}, c_{\eta}\right)$ and $\left(b_{k_{\eta+1}}, d_{\eta}\right)$ are two pairs of $\mathcal{A}_{\eta}$-equivalent ultrafilters. For simplicity, say $k_{\eta+1}=\eta+1$. We have that $\left(a_{i+1}, c_{i}\right)$ and $\left(b_{i+1}, d_{i}\right)$ are two pairs of $\mathcal{A}_{i}$-equivalent ultrafilters for each $i \in[1, \eta]$.

Put $\rho=\left\lfloor\frac{n}{3}\right\rfloor$. In view of the above, we can assume that there exist pairwise distinct ultrafilters $c_{1}, \ldots, c_{\rho-1}, c_{n}, d_{1}, \ldots, d_{\rho-1}, d_{n}$ such that the following holds:

(a) $\left(a_{1}, c_{n}\right)$ and $\left(b_{1}, d_{n}\right)$ are two pairs of $\mathcal{A}_{n}$-equivalent ultrafilters;

(b) $\left(a_{i+1}, c_{i}\right)$ and $\left(b_{i+1}, d_{i}\right)$ are two pairs of $\mathcal{A}_{i}$-equivalent ultrafilters for each $i \in[1, \rho-1]$;

(c) $\mathfrak{F} \cap\left\{c_{1}, \ldots, c_{\rho-1}, c_{n}, d_{1}, \ldots, d_{\rho-1}, d_{n}\right\}=\emptyset$, see Figure 1 .

(2) Put

$$
Z_{\rho}=\left\{a_{1}, \ldots, a_{\rho}, b_{1}, \ldots, b_{\rho}, c_{1}, \ldots, c_{\rho-1}, c_{n}, d_{1}, \ldots, d_{\rho-1}, d_{n}\right\}
$$

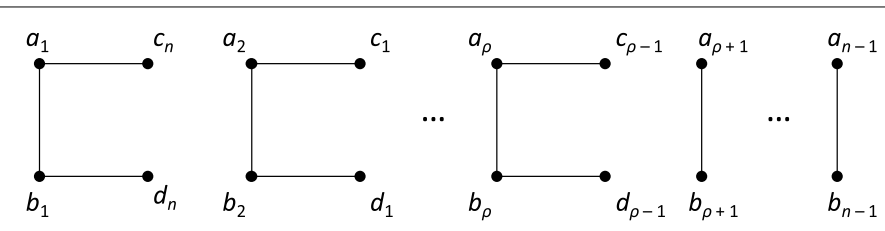

Figure 1 Ultrafilters $a_{i}, b_{i}, c_{i}$, and $d_{i}$. 


$$
\begin{aligned}
& Z_{\rho}^{\prime}=\left\{a_{1}, \ldots, a_{n-1}, b_{1}, \ldots, b_{n-1}, c_{1}, \ldots, c_{\rho-1}, c_{n}, d_{1}, \ldots, d_{\rho-1}, d_{n}\right\}, \\
& Z_{\rho}^{\prime \prime}=\left\{a_{\rho+1}, \ldots, a_{n-1}, b_{\rho+1}, \ldots, b_{n-1}\right\}, \\
& L_{\rho}=\operatorname{ker} \mathcal{A}_{\rho} \backslash Z_{\rho}^{\prime} .
\end{aligned}
$$

Clearly,

$$
\begin{aligned}
\#\left(L_{\rho}\right) \geq \frac{10}{3} n+\omega(n)-4 \cdot\left\lfloor\frac{n}{3}\right\rfloor-2\left(n-1-\left\lfloor\frac{n}{3}\right\rfloor\right) \\
=\frac{4}{3} n-2 \cdot\left\lfloor\frac{n}{3}\right\rfloor+\omega(n)+2 \geq \frac{2}{3} n+\omega(n)+2, \\
\#\left(L_{\rho}\right)-\#([\rho+1, n-1]) \geq \frac{2}{3} n+\omega(n)+2-\left(n-1-\left\lfloor\frac{n}{3}\right\rfloor\right) \\
=\left\lfloor\frac{n}{3}\right\rfloor-\frac{n}{3}+\omega(n)+3>0 .
\end{aligned}
$$

The above arguments show that the following assumption should be made: for each ultrafilter $q \in L_{\rho}$, there exists an ultrafilter $\tilde{q} \in Z_{\rho}^{\prime \prime}$ such that $q$ and $\tilde{q}$ are $\mathcal{A}_{\rho}$-equivalent ultrafilters. In general, there can be such $q$ for which the number of corresponding $\tilde{q}$ is greater than 1 . We choose in an arbitrary way only one $\tilde{q}$ for each $q \in L_{\rho}$. We obtain the mapping $f: L_{\rho} \rightarrow Z_{\rho}^{\prime \prime}, f(q)=\tilde{q}$. The map $f$ is one-to-one. (If $f\left(q_{1}\right)=f\left(q_{2}\right)$ and $q_{1} \neq q_{2}$, then $q_{1}, q_{2}$ are $\mathcal{A}_{\rho}$-similar ultrafilters, and the construction which contradicts the non-existence of ultrafilters $a_{1}^{*}, \ldots, a_{n}^{*}, b_{1}^{*}, \ldots, b_{n}^{*}$ is yielded immediately.) Put

$$
\begin{aligned}
\mathfrak{I}_{1}^{\rho}= & \left\{k \in[\rho+1, n-1] \mid \text { there exist ultrafilters } q_{k}^{a}, q_{k}^{b} \in L_{\rho}\right. \\
& \text { such that } \left.f\left(q_{k}^{a}\right)=a_{k}, f\left(q_{k}^{b}\right)=b_{k}\right\}, \\
\mathfrak{I}_{2}^{\rho}= & \left\{k \in[\rho+1, n-1] \backslash \mathfrak{I}_{1}^{\rho} \mid \text { there exists an ultrafilter } q_{k}^{*} \in L_{\rho}\right. \\
& \text { such that } \left.f\left(q_{k}^{*}\right) \in\left\{a_{k}, b_{k}\right\}\right\} .
\end{aligned}
$$

Obviously, $\mathfrak{I}_{1}^{\rho} \cap \mathfrak{I}_{2}^{\rho}=\emptyset$. Since \# $\left(L_{\rho}\right)-\#([\rho+1, n-1])>0$, we have \# $\left(\mathfrak{I}_{1}^{\rho}\right)=\tau>0$. Clearly,

$$
\#\left(\mathfrak{I}_{2}^{\rho}\right)=\#\left(L_{\rho}\right)-2 \tau \geq \frac{2}{3} n+\omega(n)+2-2 \tau .
$$

Put

$$
L_{n}=\operatorname{ker} \mathcal{A}_{n} \backslash Z_{\rho}^{\prime} .
$$

We have obtained above the estimate $\#\left(L_{\rho}\right) \geq \frac{2}{3} n+\omega(n)+2$. In exactly the same way, the following estimate can be obtained:

$$
\#\left(L_{n}\right) \geq \frac{2}{3} n+\omega(n)+2 .
$$

If there exist two $\mathcal{A}_{n}$-equivalent ultrafilters from $L_{n}$, we immediately obtain the required construction regarding the existence of ultrafilters $a_{1}^{*}, \ldots, a_{n}^{*}, b_{1}^{*}, \ldots, b_{n}^{*}$. Similarly, if an ultrafilter in $L_{n}$ has an $\mathcal{A}_{n}$-equivalent ultrafilter in $\left\{a_{1}, b_{1}, c_{1}, \ldots, c_{\rho-1}, c_{n}, d_{1}, \ldots, d_{\rho-1}, d_{n}\right\}$, then it is easy to find the corresponding ultrafilters $a_{1}^{*}, \ldots, a_{n}^{*}, b_{1}^{*}, \ldots, b_{n}^{*}$. 
We are interested in the following situation: let $q \in L_{n}$, and $q$ has an $\mathcal{A}_{n}$-equivalent ultrafilter in $\left\{a_{2}, \ldots, a_{\rho}, b_{1}, \ldots, b_{\rho}\right\}$. Let, for instance, $q$ and $a_{2}$ be $\mathcal{A}_{n}$-equivalent ultrafilters. Then let us consider $d_{1}$. For $d_{1}$ there are four possible cases:

$\langle 1\rangle d_{1} \notin \operatorname{ker} \mathcal{A}_{n} ;$

$\langle 2\rangle b_{2}, d_{1}$ are $\mathcal{A}_{n}$-equivalent ultrafilters;

$\langle 3\rangle d_{1}$ has an $\mathcal{A}_{n}$-equivalent ultrafilter in $\left\{a_{3}, \ldots, a_{\rho}, b_{3}, \ldots, b_{\rho}\right\}$;

$\langle 4\rangle d_{1}$ has an $\mathcal{A}_{n}$-equivalent ultrafilter in $Z_{\rho}^{\prime \prime}$.

In case $\langle 2\rangle$ let us consider $c_{1}$. For $c_{1}$ the possible corresponding cases are:

〈i $\quad c_{1} \notin \operatorname{ker} \mathcal{A}_{n}$;

〈ii $\quad c_{1}$ has an $\mathcal{A}_{n}$-equivalent ultrafilter in $\left\{a_{3}, \ldots, a_{\rho}, b_{3}, \ldots, b_{\rho}\right\}$;

〈iii $c_{1}$ has an $\mathcal{A}_{n}$-equivalent ultrafilter in $Z_{\rho}^{\prime \prime}$.

Consider case $\langle 3\rangle$ for $d_{1}$. Let $d_{1}, b_{3}$ be $\mathcal{A}_{n}$-equivalent ultrafilters. Let us consider $c_{2}$. For $c_{2}$ there are four possible cases:

$\langle 1\rangle c_{2} \notin \operatorname{ker} \mathcal{A}_{n} ;$

$\langle 2\rangle a_{3}, c_{2}$ are $\mathcal{A}_{n}$-equivalent ultrafilters;

$\langle 3\rangle c_{2}$ has an $\mathcal{A}_{n}$-equivalent ultrafilter in $\left\{a_{4}, \ldots, a_{\rho}, b_{2}, b_{4}, \ldots, b_{\rho}\right\}$;

$\langle 4\rangle c_{2}$ has an $\mathcal{A}_{n}$-equivalent ultrafilter in $Z_{\rho}^{\prime \prime}$.

Consider case $\langle 3\rangle$ for $c_{2}$. Let $b_{2}, c_{2}$ be $\mathcal{A}_{n}$-equivalent ultrafilters. Let us consider $c_{1}$. For $c_{1}$ the possible corresponding cases are:

ii $\quad c_{1} \notin \operatorname{ker} \mathcal{A}_{n}$;

〈ii $c_{1}$ has an $\mathcal{A}_{n}$-equivalent ultrafilter in $\left\{a_{3}, \ldots, a_{\rho}, b_{4}, \ldots, b_{\rho}\right\}$;

〈iii $c_{1}$ has an $\mathcal{A}_{n}$-equivalent ultrafilter in $Z_{\rho}^{\prime \prime}$.

Continuing these constructions in an obvious way, we find an ultrafilter

$$
q_{*} \in\left\{c_{1}, \ldots, c_{\rho-1}, d_{1}, \ldots, d_{\rho-1}\right\}
$$

such that one of the following two statements is true: (1) $q_{*} \notin \operatorname{ker} \mathcal{A}_{n}$; (2) $q_{*}$ has an $\mathcal{A}_{n^{-}}$ equivalent ultrafilter in $Z_{\rho}^{\prime \prime}$. Let us put

$$
\begin{aligned}
\alpha= & \#\left(\left\{q \in L_{n} \mid q \text { has an } \mathcal{A}_{n} \text {-equivalent ultrafilter in }\left\{a_{2}, \ldots, a_{\rho}, b_{2}, \ldots, b_{\rho}\right\}\right\}\right), \\
\beta= & \#\left(\left\{c_{1}, \ldots, c_{\rho-1}, d_{1}, \ldots, d_{\rho-1}\right\} \backslash \operatorname{ker} \mathcal{A}_{n}\right), \\
\gamma= & \#\left(\left\{q_{*} \in\left\{c_{1}, \ldots, c_{\rho-1}, d_{1}, \ldots, d_{\rho-1}\right\} \mid q_{*} \text { has an } \mathcal{A}_{n}\right.\right. \text {-equivalent } \\
& \left.\left.\quad \text { ultrafilter in } Z_{\rho}^{\prime \prime}\right\}\right) .
\end{aligned}
$$

The above constructions clearly show that $\alpha \leq \beta+\gamma$. Put

$$
\hat{L}=\left\{q \in L_{n} \cup\left\{c_{1}, \ldots c_{\rho-1}, d_{1}, \ldots, d_{\rho-1}\right\} \mid q \text { has } \mathcal{A}_{n} \text {-similar ultrafilter in } Z_{\rho}^{\prime \prime}\right\} .
$$

Clearly,

$$
\#(\hat{L}) \geq \#\left(L_{n}\right)+\gamma-\alpha \geq \frac{2}{3} n+\omega(n)+2+\beta+\gamma-\alpha \geq \frac{2}{3} n+\omega(n)+2 .
$$


So, for every ultrafilter $q \in \hat{L}$, there exists an ultrafilter $\bar{q} \in Z_{\rho}^{\prime \prime}$ such that $q$ and $\bar{q}$ are $\mathcal{A}_{n^{-}}$ similar ultrafilters. In general it can happen that for some $q$ there exist more than one corresponding $\bar{q}$. Choose arbitrarily only one ultrafilter $\bar{q}$ for each $q \in \hat{L}$. We obtain a map$\operatorname{ping} \hat{f}: \hat{L} \rightarrow Z_{\rho}^{\prime \prime}, \hat{f}(q)=\bar{q}$. Consider the corresponding map $\hat{f}: \hat{L}_{\rho} \rightarrow Z_{\rho}^{\prime \prime}$. It is one-to-one. Indeed, if $\hat{f}\left(q_{1}\right)=\hat{f}\left(q_{2}\right)$ and $q_{1} \neq q_{2}$, then $q_{1}, q_{2}$ are $\mathcal{A}_{n}$-similar ultrafilters, and the construction which contradicts the non-existence of ultrafilters $a_{1}^{*}, \ldots, a_{n}^{*}, b_{1}^{*}, \ldots, b_{n}^{*}$ is yielded immediately. Put

$$
\begin{aligned}
\hat{\mathfrak{I}}_{1}= & \left\{k \in[\rho+1, n-1] \mid \text { there exist ultrafilters } \mathfrak{q}_{k}^{a}, \mathfrak{q}_{k}^{b} \in \hat{L}\right. \\
& \text { such that } \left.\hat{f}\left(\mathfrak{q}_{k}^{a}\right)=a_{k}, \hat{f}\left(\mathfrak{q}_{k}^{b}\right)=b_{k}\right\}, \\
\hat{\mathfrak{I}}_{2}= & \left\{k \in[\rho+1, n-1] \backslash \hat{\mathfrak{I}}_{1} \mid \text { there exists an ultrafilter } \mathfrak{q}_{k}^{*} \in \hat{L}\right. \\
& \text { such that } \left.\hat{f}\left(\mathfrak{q}_{k}^{*}\right) \in\left\{a_{k}, b_{k}\right\}\right\} .
\end{aligned}
$$

Obviously, $\hat{\mathfrak{I}}_{1} \cap \hat{\mathfrak{I}}_{2}=\emptyset$. Since \#( $(\hat{L})-\#([\rho+1, n-1])>0$, we have $\#\left(\hat{\mathfrak{I}}_{1}\right)=\hat{\tau}>0$. Clearly,

$$
\#\left(\hat{\mathfrak{I}}_{2}\right)=\#(\hat{L})-2 \hat{\tau} \geq \frac{2}{3} n+\omega(n)+2-2 \hat{\tau}
$$

If $\tau \geq \hat{\tau}$, put

$$
\mathfrak{I}=\left(\hat{\mathfrak{I}}_{1} \cup \hat{\mathfrak{I}}_{2}\right) \cap \mathfrak{I}_{1}^{\rho} .
$$

If $\tau<\hat{\tau}$, put

$$
\mathfrak{I}=\left(\mathfrak{I}_{1}^{\rho} \cup \mathfrak{I}_{2}^{\rho}\right) \cap \hat{\mathfrak{I}}_{1} .
$$

Clearly,

$$
\#(\mathfrak{I}) \geq \frac{2}{3} n+\omega(n)+2-n+1+\left\lfloor\frac{n}{3}\right\rfloor>\omega(n)+2 .
$$

(3) We fix $v \in \mathfrak{I}$. A number $k \in[1, \rho]$ is called $v$-marked if the following is true: for $k=1:\left(a_{1}, d_{n}\right)$ and $\left(b_{1}, c_{n}\right)$ are pairs of $\mathcal{A}_{v}$-equivalent ultrafilters; for $k>1$ : $\left(a_{k}, d_{k-1}\right)$ and $\left(b_{k}, c_{k-1}\right)$ are pairs of $\mathcal{A}_{v}$-equivalent ultrafilters.

Put

$$
\chi_{v}=\#(\{k \in[1, \rho] \mid k \text { is a } v \text {-marked number }\}) .
$$

Our aim is to prove that

$$
\chi_{v}>\frac{\omega(n)}{2} .
$$

We have the following options:

$\langle 1\rangle$ There exist ultrafilters $q_{v}^{a}, q_{v}^{b} \in \mathfrak{I}_{1}^{\rho}$ and an ultrafilter $\mathfrak{q}_{v}^{*} \in \hat{\mathfrak{I}}_{2}$.

$\langle 2\rangle$ There exists an ultrafilter $q_{\nu}^{*} \in \mathfrak{I}_{2}^{\rho}$ and ultrafilters $\mathfrak{q}_{\nu}^{a}, \mathfrak{q}_{\nu}^{b} \in \hat{\mathfrak{I}}_{1}$.

$\langle 3\rangle$ There exist ultrafilters $q_{v}^{a}, q_{v}^{b} \in \mathfrak{I}_{1}^{\rho}$ and ultrafilters $\mathfrak{q}_{v}^{a}, \mathfrak{q}_{v}^{b} \in \hat{\mathfrak{I}}_{1}$. 
Denote $q_{v}^{*}$ by $q_{v}$. Denote $\mathfrak{q}_{v}^{*}$ by $q_{v}^{\prime}$. Choose one of two ultrafilters $q_{v}^{a}, q_{v}^{b}$ and denote it by $q_{v}$; at this step we do not consider the second ultrafilter. Choose one of two ultrafilters $\mathfrak{q}_{v}^{a}, \mathfrak{q}_{v}^{b}$ and denote it by $q_{v}^{\prime}$; at this step we do not consider the second ultrafilter. If possible, the ultrafilter $q_{v}^{\prime}$ is taken from $\hat{L} \backslash L_{n}$. Let $v=\rho+1$. We know that there exists a corresponding ultrafilter $q_{\rho+1} \in L_{\rho}$ which has an $\mathcal{A}_{\rho}$-equivalent ultrafilter in $\left\{a_{\rho+1}, b_{\rho+1}\right\}$. We also know that there exists a corresponding ultrafilter $q_{\rho+1}^{\prime} \in \hat{L}$ which has $\mathcal{A}_{n}$-equivalent ultrafilter in $\left\{a_{\rho+1}, b_{\rho+1}\right\}$.

When the number $\chi_{\rho+1}$ attains its minimal value, we must assume the following: there exist pairwise distinct ultrafilters

$$
a_{\rho+2}^{\prime}, \ldots, a_{n-1}^{\prime}, b_{\rho+2}^{\prime}, \ldots, b_{n-1}^{\prime} \in \operatorname{ker} \mathcal{A}_{\rho+1} \backslash\left(Z_{\rho}^{\prime} \cup\left\{q_{\rho+1}, q_{\rho+1}^{\prime}\right\}\right)
$$

and $\left(a_{k}, a_{k}^{\prime}\right),\left(b_{k}, b_{k}^{\prime}\right)$ are pairs of $\mathcal{A}_{\rho+1}$-equivalent ultrafilters for each $k \in[\rho+2, n-1]$. We will only consider the cases where finding ultrafilters $a_{1}^{*}, \ldots, a_{n}^{*}, b_{1}^{*}, \ldots, b_{n}^{*}$ is not immediate.

Case 1. $q_{\rho+1}^{\prime} \in L_{n}$.

Case 1-1. $q_{\rho+1}=q_{\rho+1}^{\prime}$.

We consider only two subcases of Case 1-1.

Case 1-1-1. There exists an ultrafilter $q^{*} \notin Z_{\rho}$ such that $q^{*}, q_{\rho+1}$ are $\mathcal{A}_{\rho+1}$-equivalent ultrafilters.

Case 1-1-2. There exists an ultrafilter $q^{*} \in Z_{\rho}$ such that $q^{*}, q_{\rho+1}$ are $\mathcal{A}_{\rho+1}$-equivalent ultrafilters.

Case 1-2. $q_{\rho+1} \neq q_{\rho+1}^{\prime}$.

We consider only two subcases of Case 1-2.

Case 1-2-1. $q_{\rho+1}, q_{\rho+1}^{\prime}$ are $\mathcal{A}_{\rho+1}$-equivalent ultrafilters.

Case 1-2-2. There exists an ultrafilter $q^{*} \in\left\{a_{1}, \ldots, a_{\rho}, b_{1}, \ldots, b_{\rho}\right\}$ such that $q^{*}, q_{\rho+1}$ are $\mathcal{A}_{\rho+1}$-equivalent ultrafilters.

Before we consider these cases, let us denote $\mathcal{R}_{1}=\left\{a_{1}, b_{1}, c_{n}, b_{n}\right\}$, and $\mathcal{R}_{k}=\left\{a_{k}, b_{k}, c_{k-1}\right.$, $\left.d_{k-1}\right\}$ if $k \in[2, \rho]$.

First we consider Cases 1-1-1 and 1-2-1. For the situation where the number $\chi_{\rho+1}$ attains its the minimum value, we have the following options for the set $\mathcal{R}_{1}$ :

(1) $a_{1}, b_{1}$ are $\mathcal{A}_{\rho+1}$-equivalent ultrafilters and $\#\left(\operatorname{ker} \mathcal{A}_{\rho+1} \cap \mathcal{R}_{1}\right)=2$;

(2) $a_{1}, d_{n}$ are $\mathcal{A}_{\rho+1}$-equivalent ultrafilters and $\#\left(\operatorname{ker} \mathcal{A}_{\rho+1} \cap \mathcal{R}_{1}\right)=2$;

(3) $b_{1}, c_{n}$ are $\mathcal{A}_{\rho+1}$-equivalent ultrafilters and $\#\left(\operatorname{ker} \mathcal{A}_{\rho+1} \cap \mathcal{R}_{1}\right)=2$;

(4) the number 1 is $(\rho+1)$-marked.

If $k \in[2, \rho]$, by analogy, we have the following options for the set $\mathcal{R}_{k}$ :

$\left(^{*}\right) a_{k}, b_{k}$ are $\mathcal{A}_{\rho+1}$-equivalent ultrafilters and $\#\left(\operatorname{ker} \mathcal{A}_{\rho+1} \cap \mathcal{R}_{k}\right)=2$;

$\left(^{*}\right) a_{k}, d_{k-1}$ are $\mathcal{A}_{\rho+1}$-equivalent ultrafilters and $\#\left(\operatorname{ker} \mathcal{A}_{\rho+1} \cap \mathcal{R}_{k}\right)=2$;

(3*) $b_{k}, c_{k-1}$ are $\mathcal{A}_{\rho+1}$-equivalent ultrafilters and $\#\left(\operatorname{ker} \mathcal{A}_{\rho+1} \cap \mathcal{R}_{k}\right)=2$;

$\left(4^{*}\right)$ the number $k$ is $(\rho+1)$-marked.

So we have

$$
4(n-1-\rho)+4 \cdot \chi_{\rho+1}+2\left(\rho-\chi_{\rho+1}\right)=\#\left(\operatorname{ker} \mathcal{A}_{\rho+1}\right) \geq \frac{10}{3} n+\omega(n) .
$$




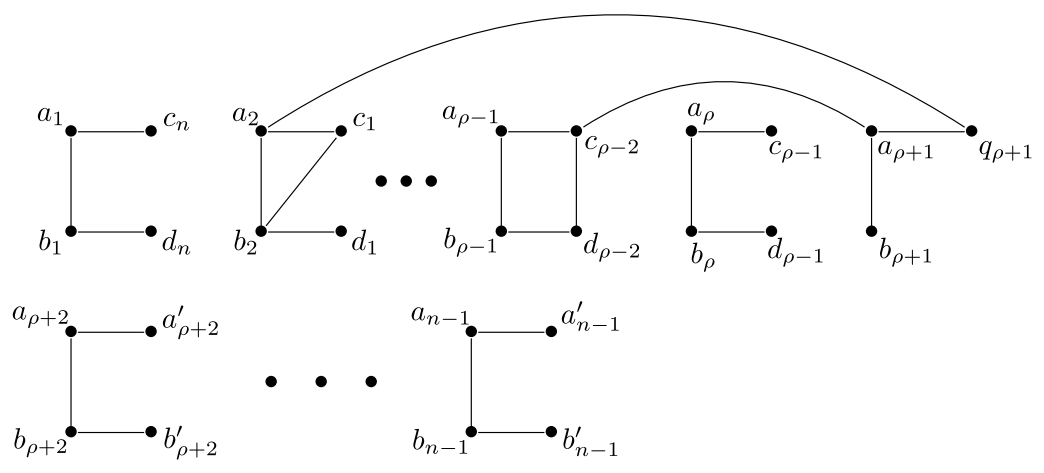

Figure 2 Illustration for Case 2.

Recall that $\rho=\left\lfloor\frac{n}{3}\right\rfloor$. Therefore we have

$$
\chi_{\rho+1}>\frac{\omega(n)}{2}+1
$$

Now consider Case 1-1-2. The situation is as follows:

$\left\langle\right.$ a) $c_{\rho-1}, q_{\rho+1}$ are $\mathcal{A}_{\rho+1}$-equivalent ultrafilters;

〈b) $a_{\rho}, d_{\rho-1}$ are $\mathcal{A}_{\rho+1}$-equivalent ultrafilters;

$\langle$ c $\rangle$ for $\mathcal{R}_{1}$ one of the options (1)-(4) is fulfilled;

$\langle$ d $\rangle$ for $\mathcal{R}_{k}$, where $k \in[2, \rho-1]$, one of the options $\left(1^{*}\right)-\left(4^{*}\right)$ is fulfilled.

Now consider Case 1-2-2. The situation is as follows: $b_{\rho}, q_{\rho+1}$ are $\mathcal{A}_{\rho+1}$-equivalent ultrafilters, and the conditions $\langle\mathrm{b}\rangle,\langle\mathrm{c}\rangle,\langle\mathrm{d}\rangle$ are fulfilled. It is clear that in Cases 1-1-2 and 1-2-2 we have

$$
\chi_{\rho+1}>\frac{\omega(n)}{2}+1
$$

It is clear that in Case 1 there may be subcases which we have not considered. But always

$$
\chi_{\rho+1}>\frac{\omega(n)}{2}+1
$$

Case 2. $q_{\rho+1}^{\prime} \in \hat{L} \backslash L_{n}$. Suppose that $q_{\rho+1}^{\prime}=c_{\rho-2}$ and $c_{\rho-2}, a_{\rho+1}$ are $\mathcal{A}_{n}$-equivalent ultrafilters. For the number $\chi_{\rho+1}$ to be minimal and the situation to be nontrivial, we assume the following:

(i) $\left(a_{\rho-1}, b_{\rho-1}\right),\left(c_{\rho-2}, d_{\rho-2}\right),\left(a_{2}, q_{\rho+1}\right),\left(b_{2}, c_{1}\right)$ are pairs of $\mathcal{A}_{\rho+1}$-equivalent ultrafilters;

(ii) $a_{\rho+1}, q_{\rho+1}$ are $\mathcal{A}_{\rho}$-equivalent ultrafilters;

(iii) $\operatorname{ker} \mathcal{A}_{\rho+1} \subset Z_{\rho}^{\prime} \cup\left\{a_{\rho+2}^{\prime}, \ldots, a_{n-1}^{\prime}, b_{\rho+2}^{\prime}, \ldots, b_{n-1}^{\prime}\right\} \cup\left\{q_{\rho+1}\right\}$, see Figure 2 .

We assume that one of the cases (1)-(4) holds for $\mathcal{R}_{1}$ and that one of the cases $\left(1^{*}\right)-\left(4^{*}\right)$ holds for $\mathcal{R}_{k}$, where $k \in[3, \rho] \backslash\{\rho-1\}$. We have

$$
4(n-1-\rho)+4 \cdot \chi_{\rho+1}+2\left(\rho-2-\chi_{\rho+1}\right)+6=\#\left(\operatorname{ker} \mathcal{A}_{\rho+1}\right) \geq \frac{10}{3} n+\omega(n) .
$$


Recall that $\rho=\left\lfloor\frac{n}{3}\right\rfloor$. Therefore we have

$$
\chi_{\rho+1}>\frac{\omega(n)}{2}
$$

Analyzing the other situations in Case 2, we come to the same conclusion: $\chi_{\rho+1}>\frac{\omega(n)}{2}$; and we can assume that if $k$ is a $(\rho+1)$-marked number, then $q_{\rho+1}^{\prime} \notin \mathcal{R}_{k}$. It is obvious that the same conclusion is true in Case 1 .

(4) It is obvious that for each $v \in \mathfrak{I}$ we have $\chi_{v}>\frac{\omega(n)}{2}$, and $q_{k}^{\prime} \notin \mathcal{R}_{k}$ if $k$ is a $v$-marked number. We know that

$$
\omega(n) \geq \sqrt{\frac{2 n}{3}}, \quad \#(\mathfrak{I})>\omega(n)+2, \quad \rho=\left\lfloor\frac{n}{3}\right\rfloor
$$

Therefore we have

$$
\frac{\omega(n)}{2} \cdot \#(\mathfrak{I})>\frac{\omega(n)}{2} \cdot(\omega(n)+2)>\rho .
$$

Therefore there exist distinct numbers $v_{1}, v_{2} \in \mathfrak{I}$ and $k_{0} \in[1, \rho]$ such that $k_{0}$ is a $v_{1}$-marked number and $v_{2}$-marked number. Let $v_{1}=\rho+1, v_{2}=\rho+2$. Consider the ultrafilters $q_{\rho+1}$, $q_{\rho+2}^{\prime}$. If $q_{\rho+1} \neq q_{\rho+2}^{\prime}$, put $z_{\rho+1}=q_{\rho+1}, z_{\rho+2}^{\prime}=q_{\rho+2}^{\prime}$. Let $q_{\rho+1}=q_{\rho+2}^{\prime}$. There are two possible cases.

I. There exist the ultrafilters $q_{\rho+1}^{a}, q_{\rho+1}^{b}$, and assume that $q_{\rho+1}=q_{\rho+1}^{b}$. Put $z_{\rho+1}=q_{\rho+1}^{a}, z_{\rho+2}^{\prime}=$ $q_{\rho+2}^{\prime}$.

II. The ultrafilters $q_{\rho+1}^{a}, q_{\rho+1}^{b}$ do not exist. Then there exist the ultrafilters $\mathfrak{q}_{\rho+1}^{a}, \mathfrak{q}_{\rho+1}^{b}$, and assume that $q_{\rho+1}^{\prime}=\mathfrak{q}_{\rho+1}^{b}$. Put $z_{\rho+2}=q_{\rho+2}$. If $q_{\rho+1}^{\prime} \neq z_{\rho+2}$, put $z_{\rho+1}^{\prime}=q_{\rho+1}^{\prime}$. Otherwise we have $\mathfrak{q}_{\rho+1}^{a} \in L_{n}$ since $q_{\rho+1}^{\prime}=q_{\rho+2} \in L_{n}$ (see in the part (3) of our proof how we have chosen the ultrafilter $q_{v}^{\prime}$ ); and put $z_{\rho+1}^{\prime}=\mathfrak{q}_{\rho+1}^{a}$.

Thus, we consider either the pair of ultrafilters $z_{\rho+1}, z_{\rho+2}^{\prime}$, or the pair of ultrafilters $z_{\rho+1}^{\prime}$, $z_{\rho+2}$. These two pairs have the same properties. We will consider the pair $z_{\rho+1}, z_{\rho+2}^{\prime}$. We have the following:

$1^{\circ} z_{\rho+1}$ has an $\mathcal{A}_{\rho}$-equivalent ultrafilter in $\left\{a_{\rho+1}, b_{\rho+1}\right\}$;

$2^{\circ} z_{\rho+2}^{\prime}$ has an $\mathcal{A}_{n}$-equivalent ultrafilter in $\left\{a_{\rho+2}, b_{\rho+2}\right\}$;

$3^{\circ} z_{\rho+1} \neq z_{\rho+2}^{\prime}$;

$4^{\circ} z_{\rho+1} \notin Z_{\rho}^{\prime}$;

$5^{\circ} z_{\rho+2}^{\prime} \notin \mathfrak{F} \cup \mathcal{R}_{k_{0}}$.

Suppose that $a_{\rho+1}$ and $z_{\rho+1}$ are $\mathcal{A}_{\rho}$-equivalent ultrafilters, $a_{\rho+2}$ and $z_{\rho+2}^{\prime}$ are $\mathcal{A}_{n}$-equivalent ultrafilters, and $k_{0}=3$. It is possible that

$$
z_{\rho+2}^{\prime} \in\left\{c_{3}, \ldots, c_{\rho-1}, d_{3}, \ldots, d_{\rho-1}\right\}
$$

Suppose that

$$
z_{\rho+2}^{\prime} \notin\left\{d_{3}, \ldots, d_{\rho-1}\right\}
$$

Now it is easy to construct the corresponding ultrafilters $a_{1}^{*}, \ldots, a_{n}^{*}, b_{1}^{*}, \ldots, b_{n}^{*}$. Let us list them in pairs: $\left(a_{1}^{*}, b_{1}^{*}\right)=\left(a_{1}, b_{1}\right),\left(a_{2}^{*}, b_{2}^{*}\right)=\left(a_{2}, b_{2}\right),\left(a_{3}^{*}, b_{3}^{*}\right)=\left(b_{4}, d_{3}\right), \ldots,\left(a_{\rho-1}^{*}, b_{\rho-1}^{*}\right)=$ 


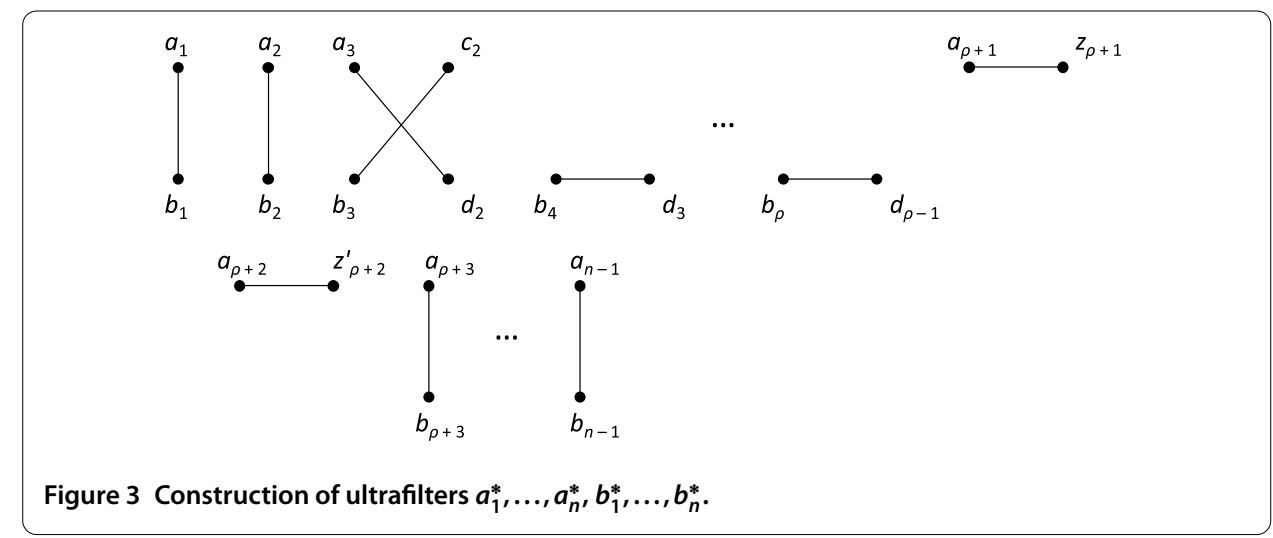

$\left(b_{\rho}, d_{\rho-1}\right),\left(a_{\rho}^{*}, b_{\rho}^{*}\right)=\left(a_{\rho+1}, z_{\rho+1}\right),\left(a_{\rho+1}^{*}, b_{\rho+1}^{*}\right)=\left(a_{3}, d_{2}\right),\left(a_{\rho+2}^{*}, b_{\rho+2}^{*}\right)=\left(b_{3}, c_{2}\right),\left(a_{\rho+3}^{*}, b_{\rho+3}^{*}\right)=$ $\left(a_{\rho+3}, b_{\rho+3}\right), \ldots,\left(a_{n-1}^{*}, b_{n-1}^{*}\right)=\left(a_{n-1}, b_{n-1}\right),\left(a_{n}^{*}, b_{n}^{*}\right)=\left(a_{\rho+2}, z_{\rho+2}^{\prime}\right)$, see Figure 3 .

\section{Combinatorial theorems}

In this section we consider for each $n \in \mathbb{N}^{+}$a matrix $\mathfrak{M}(n)$ which has $n$ rows and $\aleph_{0}$ columns. We denote by $\alpha_{i}^{k}$ the element of $\mathfrak{M}(n)$ in the $i$ th row and the $k$ th column. The following holds:

(1) $\alpha_{i}^{k} \in \mathbb{N}$;

(2) for any $\alpha_{i}^{k}>0$, there exists $\alpha_{i}^{k^{\prime}}$ such that $\alpha_{i}^{k}=\alpha_{i}^{k^{\prime}}$ and $k \neq k^{\prime}$.

We denote by $w(\mathfrak{M}(n), i)$ the number of nonzero elements in the $i$ th row of $\mathfrak{M}(n)$. It is clear that

$$
0 \leq w(\mathfrak{M}(n), i) \leq \aleph_{0}
$$

Definition 4.1 A matrix $\mathfrak{M}(n)$ is said to be saturated if there exist pairwise distinct natural numbers $k_{1}, k_{1}^{\prime}, \ldots, k_{n}, k_{n}^{\prime}$ such that $\alpha_{i}^{k_{i}}=\alpha_{i}^{k_{i}^{\prime}}>0$ for each $i \in[1, n]$.

Definition 4.2 For each $n \in \mathbb{N}^{+}$, denote by $\mathfrak{v}^{\prime}(n)$ the minimal natural number such that if for some matrix $\mathfrak{M}(n)$ we have $w(\mathfrak{M}(n), i) \geq \mathfrak{v}^{\prime}(n)$ for each $i \in[1, n]$, then $\mathfrak{M}(n)$ is saturated.

We suppose that $\mathfrak{v}^{\prime}(n) \in \mathbb{N}^{+}$since, obviously, $\mathfrak{v}^{\prime}(n)<\aleph_{0}$.

It is easy to prove that $\mathfrak{v}(n)=\mathfrak{v}^{\prime}(n)$. Therefore, by Theorem 2.1, the following theorem is true.

Theorem 4.3 If for some matrix $\mathfrak{M}(n)$ we have

$$
w(\mathfrak{M}(n), i) \geq \frac{10}{3} n+\sqrt{\frac{2 n}{3}}
$$

for each $i \in[1, n]$, then $\mathfrak{M}(n)$ is saturated.

The following theorem is a particular case of the well-known theorem of Ramsey [12].

Theorem 4.4 Consider a set $S, \#(S)=n \in \mathbb{N}^{+}$, and let $T$ be the family of all two-element subsets of $S$. We divide $T$ into two disjoint sub-families $T_{1}, T_{2}$. Fix a natural number $\mu \geq 2$. 
We claim that there exists the minimal number $R(\mu) \in \mathbb{N}^{+}$such that if $n \geq R(\mu)$, then there exists a set $S^{\prime} \subset S, \#\left(S^{\prime}\right)=\mu$, and either all two-element subsets of $S^{\prime}$ belong to $T_{1}$ or they all belong to $T_{2}$.

In the formulation of the following theorem, we use the number $R(\mu)$ from Theorem 4.4.

Theorem 4.5 Consider a matrix $\mathfrak{M}(n)$, and fix a natural number $\mu \geq 2$. Let

$$
w(\mathfrak{M}(n), i) \geq \frac{10}{3} n+\sqrt{\frac{2 n}{3}}
$$

for any $i \in[1, n]$, and $n \geq R(\mu)$. Then

(1) there exist pairwise distinct natural numbers

$$
k_{1}, k_{1}^{\prime}, \ldots, k_{n}, k_{n}^{\prime}
$$

such that $\alpha_{i}^{k_{i}}=\alpha_{i}^{k_{i}^{\prime}}>0$ and $k_{i}<k^{\prime}$ for each $i \in[1, n]$;

(2) there exists a family of segments

$$
D \subset\left\{\left[k_{i}, k_{i}^{\prime}\right]\right\}_{i \leq n}
$$

$\#(D)=\mu$, and one of the following two cases holds;

(a) if $I_{1}, I_{2} \in D$ are distinct, then $I_{1} \cap I_{2}=\emptyset$;

(b) $\cap D \neq \emptyset$. $^{\mathrm{c}}$

Proof Let us use the notation of Theorem 4.4. By Theorem 4.3 there exists a corresponding family of segments

$$
S=\left\{\left[k_{i}, k_{i}^{\prime}\right]\right\}_{i \leq n}
$$

Let $T$ be the family of all subsets of $S$ with the exact two elements. Divide $T$ into two disjoint sub-families $T_{1}, T_{2}$. Let $T_{1}$ be the family of pairs of disjoint segments. Let $T_{2}$ be the family of pairs of distinct joint segments. By Theorem 4.4 there exists a family $D \subset S$ such that $\#(D)=\mu$ and all pairs of distinct segments from $D$ belong either to $T_{1}$ or to $T_{2}$. If all pairs of distinct segments belong to $T_{2}$, then it is easy to see that $\cap D \neq \emptyset$.

Remark 4.6 The following well-known result is given, for example, in [13]:

$$
R(\mu) \leq\left(\begin{array}{c}
2 \mu-2 \\
\mu-1
\end{array}\right) .
$$

Therefore Theorem 4.5 is true if the condition $n \geq R(\mu)$ will be exchanged by $n \geq\left(\begin{array}{c}2 \mu-2 \\ \mu-1\end{array}\right)$.

\section{Countable families of $\sigma$-algebras}

In the first nine subsections we present facts from [1] and [2].

Definition 5.1 A point $a \in \beta X$ is said to be irregular if for any countable sequence of sets $M_{1}, \ldots, M_{k}, \ldots \subset \beta X$ such that $a \notin \bar{M}_{k}$ for all $k$, we have $a \notin \overline{\cup M_{k}}$. 
Since a point of $\beta X$ is an ultrafilter on $X$ and, vice versa, an ultrafilter on $X$ is a point of $\beta X$, we will also call an irregular point an irregular ultrafilter. All points of $X$ are irregular.

Definition 5.2 An algebra $\mathcal{A}$ is said to be simple if there exists $Z \subseteq \beta X$ such that:

(1) $\#(Z) \leq \aleph_{0}$

(2) if $Z \neq \emptyset$, all points of $Z$ are irregular;

(3) $\operatorname{ker} \mathcal{A} \subseteq \bar{Z}$.

The proof of the following theorem is in [2], Chapter 17.

Theorem 5.3 Let $\mathcal{A}_{1}, \ldots, \mathcal{A}_{k}, \ldots$ and $\mathcal{B}_{1}, \ldots, \mathcal{B}_{k}, \ldots$ be two countable families of $\sigma$-algebras. Let all algebras $\mathcal{A}_{k}$ be simple, and among the algebras $\mathcal{B}_{k}$ let there be no simple algebras. Then there exist pairwise disjoint sets $W, U_{1}, \ldots, U_{k}, \ldots, V_{1}, \ldots, V_{k}, \ldots$ such that:

(1) $\operatorname{ker} \mathcal{A}_{k} \subseteq \bar{W}$ for each $k$;

(2) for each $k \in \mathbb{N}^{+}$, the following holds: if a set $Q$ contains one of the two sets $U_{k}, V_{k}$ and intersection with the other set is empty, then $Q \notin \mathcal{B}_{k}$.

Remark 5.4 The Gitik-Shelah theorem is essentially used in the proof of Theorem 5.3. Under the assumption that the continuum hypothesis $\left(\aleph_{1}=2^{\aleph_{0}}\right)$ is true, the proof of Theorem 5.3 essentially uses not the nontrivial Gitik-Shelah theorem but the rather simple Alaoglu-Erdös theorem.

Definition 5.5 The set $\{a \in \operatorname{ker} \mathcal{A} \mid a$ is an irregular point $\}$ is called the spectrum of an algebra $\mathcal{A}$ and is denoted $s p \mathcal{A}$.

It is clear that if $\mathcal{A}$ is a simple algebra, then $\#(s p \mathcal{A}) \leq \aleph_{0}$.

The proof of the lemma below is in [2], Chapter 7.

Lemma 5.6 If $\mathcal{A}$ is a simple $\sigma$-algebra, then $\operatorname{ker} \mathcal{A} \subseteq \overline{\operatorname{sp} \mathcal{A}}$.

The proof of the lemma below is in [2], Chapter 7.

Lemma 5.7 If $\mathcal{A}$ is a simple $\sigma$-algebra and $a \in \operatorname{sp} \mathcal{A}$, then

$$
\{b \in \operatorname{sp} \mathcal{A} \mid a \text { is } \mathcal{A} \text {-equivalent to } b\} \neq \emptyset .
$$

Remark 5.8 If an $\omega$-saturated algebra $\mathcal{A}$ is a $\sigma$-algebra, then $\mathcal{A}$ is simple and $\operatorname{ker} \mathcal{A}=s p \mathcal{A}$.

The proof of the following lemma is easily derived from Lemma 5.7 and arguments in Remark 1.13.

Lemma 5.9 Let $\mathcal{A}$ be a simple but not $\omega$-saturated $\sigma$-algebra $\mathcal{A}$ and let $v \in \mathbb{N}^{+}$. We can construct an $\omega$-saturated $\sigma$-algebra $\mathcal{A}^{\prime}$ such that $\operatorname{ker} \mathcal{A}^{\prime} \subset \operatorname{sp} \mathcal{A}, \#\left(\operatorname{ker} \mathcal{A}^{\prime}\right) \geq v$, and two ultrafilters are $\mathcal{A}^{\prime}$-equivalent if and only if they are $\mathcal{A}$-equivalent. ${ }^{\mathrm{d}}$

Proof of Theorem 2.4 Consider a sequence of integers $n_{0}=0<n_{1}<n_{2}<\cdots<n_{m}<\cdots$. Construct the function $\varphi: \mathbb{N}^{+} \rightarrow \mathbb{N}^{+}$as follows: if $k \in\left[n_{m-1}+1, n_{m}\right]$, where $m \in \mathbb{N}^{+}$, then

$$
\varphi(k)=4 \cdot n_{m-1}+\left\lceil\frac{10}{3}\left(n_{m}-n_{m-1}\right)+\sqrt{\frac{2\left(n_{m}-n_{m-1}\right)}{3}}\right\rceil .
$$


We can choose numbers $n_{1}, n_{2}, \ldots, n_{m}, \ldots$ such that condition (1) of our theorem is true. By Theorem 5.3 and Lemma 5.9 we can suppose that all algebras $\mathcal{A}_{k}$ are $\omega$-saturated $\sigma$ algebras. Put $\mathcal{A}_{k}^{\prime}=\mathcal{A}_{k}$ if $k \in\left[1, n_{1}\right]$. By Theorem 2.1 there exists a set of pairwise distinct irregular ultrafilters $G_{1}=\left\{s_{1}, t_{1}, \ldots, s_{n_{1}}, t_{n_{1}}\right\}$, and $s_{k}, t_{k}$ are $\mathcal{A}_{k}^{\prime}$-equivalent ultrafilters for each $k \in\left[1, n_{1}\right]$. Let $k \in\left[n_{1}+1, n_{2}\right]$ and

$$
E_{k}=\left\{a \in \operatorname{ker} \mathcal{A}_{k} \backslash G_{1} \mid a \text { has } A_{k} \text {-equivalent ultrafilter in } \operatorname{ker} \mathcal{A}_{k} \backslash G_{1}\right\}
$$

We can construct (see Remark 1.13) $\omega$-saturated $\sigma$-algebra $\mathcal{A}_{k}^{\prime}$ and

(1) $\operatorname{ker} \mathcal{A}_{k}^{\prime}=E_{k}$;

(2) two ultrafilters are $\mathcal{A}_{k}^{\prime}$-equivalent if and only if they are $\mathcal{A}_{k}$-equivalent.

In view of Remark 1.12, $\mathcal{A}_{k}^{\prime} \supseteq \mathcal{A}_{k}$. It is clear that

$$
\#\left(\operatorname{ker} \mathcal{A}_{k}^{\prime}\right) \geq\left\lceil\frac{10}{3}\left(n_{2}-n_{1}\right)+\sqrt{\frac{2\left(n_{2}-n_{1}\right)}{3}}\right\rceil .
$$

By Theorem 2.1 there exist pairwise distinct irregular ultrafilters $s_{n_{1}+1}, t_{n_{1}+1}, \ldots, s_{n_{2}}, t_{n_{2}}$, and $s_{k}, t_{k}$ are $\mathcal{A}_{k}^{\prime}$-equivalent ultrafilters for each $k \in\left[n_{1}+1, n_{2}\right]$. Put

$$
G_{2}=\left\{s_{1}, t_{1}, \ldots, s_{n_{2}}, t_{n_{2}}\right\}
$$

It is clear that $\#\left(G_{2}\right)=2 n_{2}$. Consider algebras $\mathcal{A}_{n_{2}+1}, \ldots, \mathcal{A}_{n_{3}}$. We can construct corresponding algebras $\mathcal{A}_{n_{2}+1}^{\prime}, \ldots, \mathcal{A}_{n_{3}}^{\prime}$, and

$$
\begin{aligned}
& \operatorname{ker} \mathcal{A}_{k}^{\prime} \cap G_{2}=\emptyset \\
& \#\left(\operatorname{ker} \mathcal{A}_{k}^{\prime}\right) \geq\left\lceil\frac{10}{3}\left(n_{3}-n_{2}\right)+\sqrt{\frac{2\left(n_{3}-n_{2}\right)}{3}}\right\rceil
\end{aligned}
$$

for each $k \in\left[n_{2}+1, n_{3}\right]$ and so on. Further, we consider algebras $\mathcal{A}_{n_{3}+1}, \ldots, \mathcal{A}_{n_{4}}$ and so on. So we can construct pairwise distinct irregular ultrafilters

$$
s_{1}, t_{1}, \ldots, s_{k}, t_{k}, \ldots
$$

such that $s_{k}, t_{k}$ are $\mathcal{A}_{k}$-equivalent ultrafilters for each $k \in \mathbb{N}^{+}$. We can construct a corresponding family of sets $\left\{U_{k}^{1}, U_{k}^{2}\right\}_{k \in \mathbb{N}^{+}}$(see Definition 1.3).

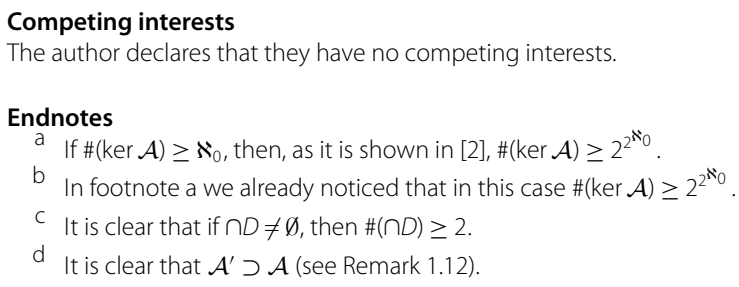


References

1. Grinblat, LŠ: On sets not belonging to algebras of subsets. Mem. Am. Math. Soc. 100, 480 (1992)

2. Grinblat, LŠ: Algebras of Sets and Combinatorics. Translations of Mathematical Monographs, vol. 214. Am. Math. Soc., Providence (2002)

3. Grinblat, LŠ: Theorems on sets not belonging to algebras. Electron. Res. Announc. Am. Math. Soc. 10, 51-57 (2004)

4. Grinblat, LŠ: On sets not belonging to algebras. J. Symb. Log. 72, 483-500 (2007)

5. Grinblat, LŠ: Theorems with uniform conditions on sets not belonging to algebras. In: Topology Proceedings, vol. 33, pp. 361-380 (2009)

6. Grinblat, LŠ: Finite and countable families of algebras of sets. Math. Res. Lett. 17, 613-623 (2010)

7. Grinblat, LŠ: Limitations on representing $\mathcal{P}(X)$ as a union of proper subalgebras. Proc. Am. Math. Soc. 143, 859-868 (2015)

8. Erdös, P: Some remarks on set theory. Proc. Am. Math. Soc. 1, 127-141 (1950)

9. Ulam, S: Zur Masstheorie in der allgemeinen Mengenlehre. Fundam. Math. 16, 140-150 (1930)

10. Gitik, M, Shelah, S: Forcing with ideals and simple forcing notions. Isr. J. Math. 68, 129-160 (1989)

11. Gleason, AM: Projective topological spaces. III. J. Math. 2, 482-489 (1958)

12. Ramsey, FP: On a problem of formal logic. Proc. Lond. Math. Soc.. 30, 264-286 (1930)

13. van Lint, JH, Wilson, RM: A Course in Combinatorics. Cambridge University Press, Cambridge (1992)

\section{Submit your manuscript to a SpringerOpen ${ }^{\circ}$ journal and benefit from:}

- Convenient online submission

Rigorous peer review

- Immediate publication on acceptance

- Open access: articles freely available online

- High visibility within the field

- Retaining the copyright to your article 